\title{
NEW COEFFICIENTS OF THE MINORITY CARRIER LIFETIME AND BANDGAP NARROWING MODELS IN THE TRANSPARENT EMITTER OF THIN FILM SILICON SOLAR CELLS
}

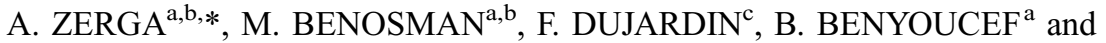 \\ J-P. CHARLES ${ }^{\mathrm{b}}$ \\ ${ }^{a}$ LMER, Départment de Physique, Faculté des Sciences, Université de Tlemcen, \\ BP: 119 Tlemcen 13000, Algerie; ${ }^{\mathrm{b}}$ C2EA, Laboratoire M.O.P.S. (CNRS-Supélec) 2 rue Edouard Belin, \\ 57070 Metz, France; ${ }^{\mathrm{c}}$ LTMC, Institut de Physique, 1 Boulevard ARAGO 57070 Metz, France
}

(Received 3 June 2001; In final form 13 August 2001)

\begin{abstract}
In this study we have determined new coefficients for the physical model describing the band-gap narrowing and the minority carriers lifetime. This was accomplished according to the doping level of the thin emitter. This model allows us to take into account both the effects of the heavy doping and the majority carrier degeneration for the very high level of doping. The results we obtain by the corrected model are in good agreement with those reported in the literature and in different experiments. They show us the possibility of accurately evaluating the performances for the $n^{+} p$ silicon solar cell. This model is then used to introduce a new concept for the thin layer emitter, called transparent emitter.
\end{abstract}

Keywords: Heavy doping effects; Transparent emitter; Silicon solar cell; Modeling-thin layer

\section{INTRODUCTION}

In opto-electronics, most semiconductor devices (junctions, diodes, transistors, solar cells. ..) include some heavily doped regions. These regions constitute an actual reservoir for the conduction current.

Generally, the application of these regions is motivated both by the use of a thin layer with low resistivity and by the possibility of enhancing the ohmicity of the metal/semiconductor contact (thanks to a difference in ohmic levels).

For the low levels of doping, the edges of the band structure are delimited and the width of the band gap is constant. When the level of doping increases, some changes affect the structure band, such as reduction of the width of the band-gap, enlargement of impurity levels in impurity bands and the apparition of $\langle\langle$ tails $\rangle\rangle$ of conduction and valence bands [1].

\footnotetext{
* Corresponding author. Tel.: 213432003 30; Fax: 213432727 89; E-mail: a_zerga@mail.univ-tlemcen.dz
} 
These changes are more complex if the doping level depends on the position. The band structures therefore become dependent of the position. This new shape of band structures poses severe problems for an adequate understanding of the minority carrier transport and recombination physics.

In the literature, many researches were published [2-4] to raise these problems and to find a reliable concept that will allow us accurate simulation for the solar cell with a highly doped emitter. Although the majority of these studies provide a good description of the heavy doping effects. The results they produce are however quite different from those of the experiments, particularly into very high doping levels.

In this fact, we numerically determined the new coefficients of the physical model describing the band-gap narrowing and the minority carriers lifetime according to the level of the doping thin layer, for different doping profiles. This model takes into consideration the effects of the majority carrier degeneration for the doping which exceeds $10^{19} \mathrm{~cm}^{-3}$ in the vicinity of the front surface. The results we obtain are described in this study and are compared to those of the literature. They show that it is possible to anticipate the performance of the considered solar cell with great accuracy.

\section{EFFECTS OF HEAVY DOPING}

To the low level of injection, the saturation current of minority carrier in homogeneous emitter of type $n$ is given by the following equation:

$$
I_{o}=q \frac{n_{\mathrm{io}}^{2}}{N_{D}} \cdot \frac{D_{p}}{L_{p}}
$$

where $n_{\mathrm{io}}$ is intrinsic concentration to the thermal equilibrium, $N_{D}$ is the concentration of donors and $\left(D_{p}, L_{p}\right)$ are respectively the coefficient and length diffusion of minority carrier.

Whereas, the open circuit voltage determined from the principle of superposition is:

$$
V_{\mathrm{oc}}=\frac{\mathrm{k} T}{q} \ln \left(\frac{I_{\mathrm{sc}}}{I_{o}}+1\right)
$$

by substituting Eq. (1) into Eq. (2), the open circuit voltage becomes:

$$
V_{\mathrm{oc}}=\frac{\mathrm{k} T}{q} \ln \left(\frac{1}{q} \cdot \frac{I_{\mathrm{sc}}}{n_{\mathrm{io}}^{2}} \cdot \frac{L_{p}}{D_{p}} \cdot N_{D}+1\right)
$$

Relating to Eq. (3), we note that the open circuit voltage $V_{\text {oc }}$ increases with the level of doping $N_{D}$. Unfortunately, in experiment it is not satisfied in high levels of doping $\left(N_{D}>10^{17} \mathrm{~cm}^{-3}\right)$. In this case, and as it is clearly shown in Figure 1, the open circuit voltage $V_{\text {oc }}$ starts to decrease, at a level doping of $10^{17} \mathrm{~cm}^{-3}$.

The difference observed between the theory and the experiment of the open circuit voltage values is due to the negligence of some physical mechanisms inherent to the heavy doping $[6,7]$. These mechanisms can be classified in two categories [8]: the band-gap narrowing and the change of the inter-bands rate transitions. 


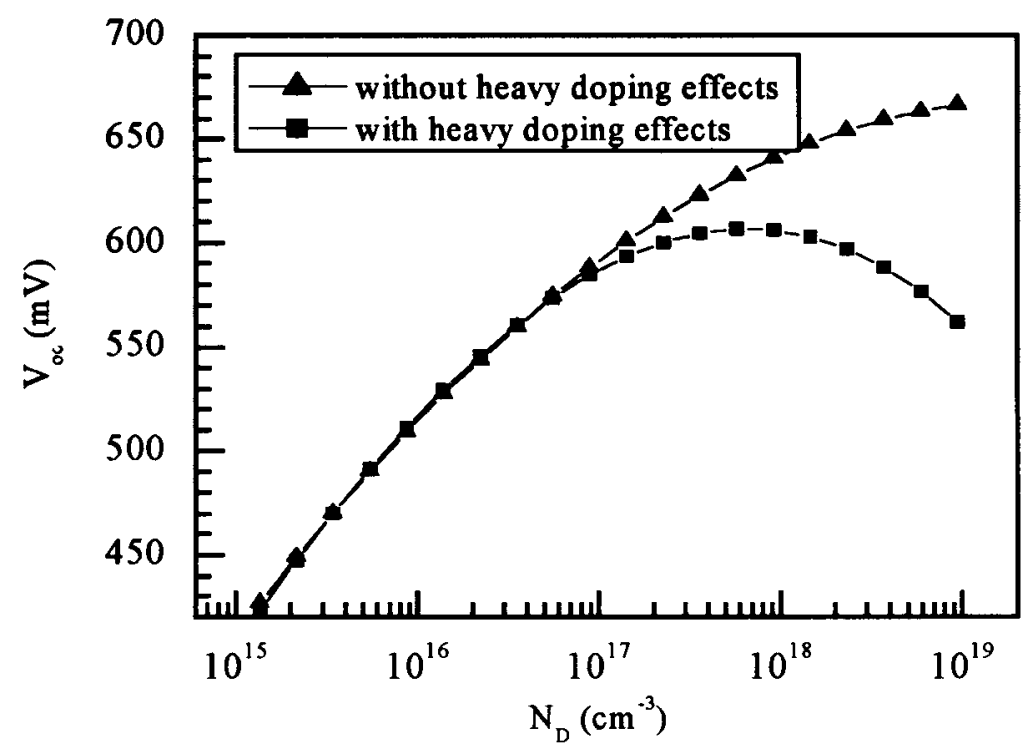

FIGURE 1 Variation of the open circuit voltage of the $n^{+} p$ silicon solar cells with respect to the level doping $N_{D}$ [5].

\subsection{Band-Gap Narrowing}

Physically, the band-gap narrowing is the consequence of the distortion of energy bands boundary, which results mainly from an interaction between donor atoms and free carriers. In the heavy doping regions, these mechanisms can be represented by three different cases [8]:

- The limits of the valence and the conduction bands develop the $\langle\langle$ tails $\rangle\rangle$ and penetrate in the band gap.

- If the concentration of donor impurities is high, the energy level of these impurities broadens into an energy band, which rides with the band of conduction.

- In very high impurity concentration, a spatial variation of the bandgap is induced. It is due to the distortion of the crystalline network.

All these mechanisms affect both the spectral response and the open circuit voltage of the silicon solar cells. They are inserted in equations describing the minority carrier transport in highly doped regions, by an effective intrinsic concentration $n_{\text {ie }}$. This concentration, depends exponentially on the band-gap narrowing $E_{g}$ according to the following relation [10]

$$
n_{\mathrm{ie}}^{2}(X)=n_{\mathrm{io}}^{2} \exp \left(\frac{\Delta E_{g}(x)}{\mathrm{k} T}\right)
$$

Most models of the band-gap narrowing are developed from the applied measures for the highly doped regions of semiconductor devices.

For the doping levels greater than $10^{17} \mathrm{~cm}^{-3}$, Slotbooms et al. (1976) [6] suggest a semiempiric model. This model is adopted in many works and describes $E_{g}$ according to the total doping level $N=N_{D}+N_{A}$ by

$$
\Delta E_{g}(\mathrm{mV})=9\left[\ln \left(\frac{N}{10^{17}}\right)+\sqrt{\left[\ln ^{2}\left(\frac{N}{10^{17}}\right)+0.5\right]}\right]
$$



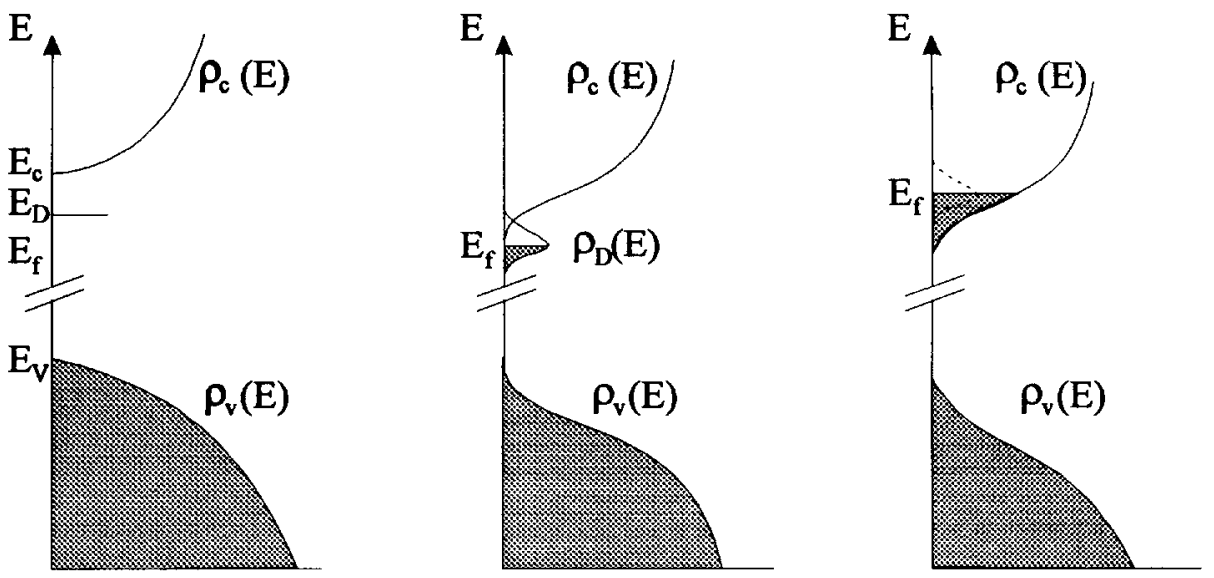

\section{Doping level}

FIGURE 2 The changes of the band structure in highly doping semiconductor [9].

Lanyon (1980) [11] has presented a new model elaborated from a theoretical calculation. This calculus is based on the free carrier screening. At the ambient temperature, $E_{g}$ is given by:

$$
\Delta E_{g}=255\left(\frac{n}{10^{20}}\right)^{0.5}
$$

Del Alamo and Swanson (1987) [12] have considered an effective band-gap narrowing $E_{\text {geff }}$ that also allows to take into account the majority carrier degeneration effects. $E_{\text {geff }}$ and $E_{g}$ are related by the following equation:

$$
\Delta E_{\mathrm{geff}}=\Delta E_{g}+\ln \left[\frac{F_{1 / 2}\left(E_{\mathrm{Fn}}-E_{C}\right)}{\exp \left(E_{\mathrm{Fn}}-E_{C}\right)}\right]
$$

where $F_{1 / 2}$ is the integral of Fermi-Dirac with $1 / 2$ order and $\left(E_{\mathrm{Fn}}-E_{C}\right)$ is the Fermi quasi-level of the majority carriers. Del Alamo et al. (1987) [12] developed this model and proposed the following semi-empirical expression for a doping level greater than $10^{17} \mathrm{~cm}^{-3}$ :

$$
\Delta E_{\mathrm{geff}}(\mathrm{eV})=A \ln \frac{N_{\mathrm{D}}}{B}
$$

where $A=18.7 \times 10^{-3} \mathrm{eV}$ and $B=7 \times 10^{17} \mathrm{~cm}^{-3}$. The graphic representation of this model shows the existence of an important difference with the experiments, particularly for doping included between $10^{19} \mathrm{~cm}^{-3}$ and $10^{20} \mathrm{~cm}^{-3}$ (effects of degeneration). In 1997, Green [3] developed this model too while bringing corrections to coefficients $A\left(14 \times 10^{-3} \mathrm{eV}\right)$ and $B\left(1.4 \times 10^{17} \mathrm{~cm}^{-3}\right)$. These new coefficients produce a correction only on a part of the level doping as shown in Figure 3. In our study, we developed a numerical program to fit 


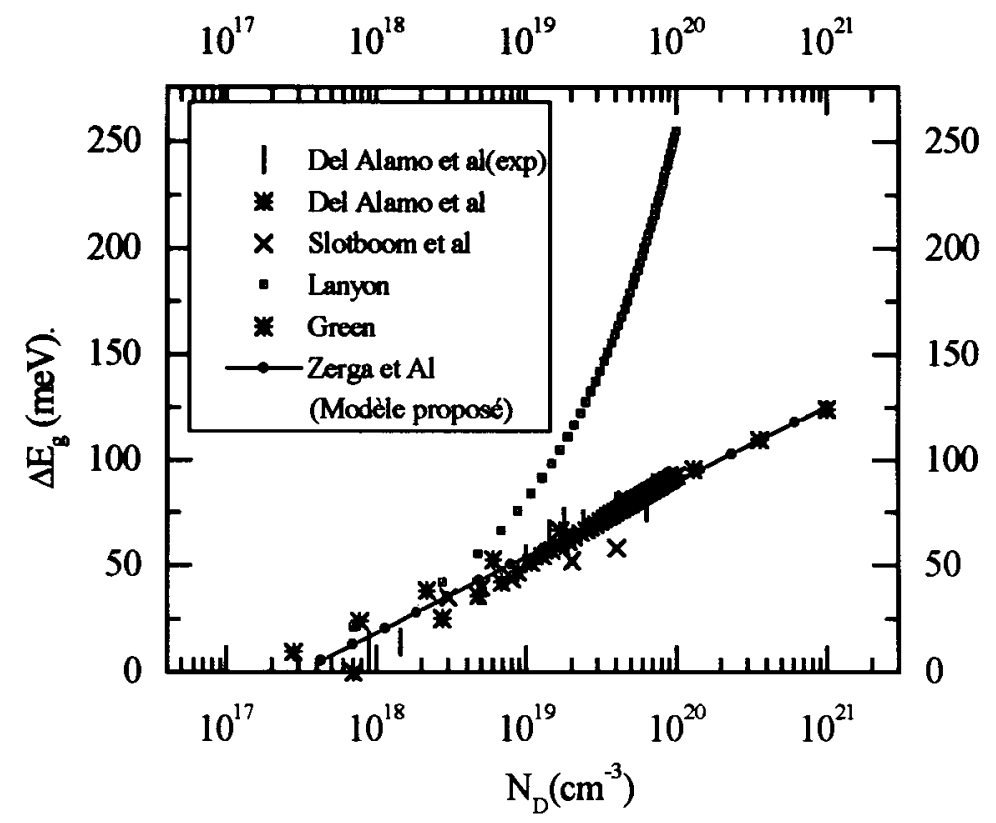

FIGURE 3 The band-gap narrowing $\Delta E_{g}$ according to the donor's concentration $N_{D}$.

the experimental results and adjust these coefficients. This was achieved by using a minimization criterion of tolerance between the theoretical and experimental results, in an interval of doping from $10^{17} \mathrm{~cm}^{-3}$ up to $10^{21} \mathrm{~cm}^{-3}$. The new coefficients we obtain by this new program, are respectively, $A=15.5 \times 10^{-3} \mathrm{eV}$ and $B=3 \times 10^{17} \mathrm{~cm}^{-3}$. Figure 3 , depicts the results of this study.

\subsection{Change of the Transitions Inter-Bands Rate}

To the high doping levels, the minority carrier lifetime is induced mainly from the band to band transition with collision of Auger type. It's inversely proportional to the square of the impurity concentration [13]. It represents an important impact on the performances of silicon solar cells where the short circuit current decreases and the saturation current increases [14]. In the last decade, many methods have been proposed to evaluate the minority carrier lifetime relating to the doping level and to explain the difference between the theory and the experiment of the open circuit voltage.

Generally, the thin emitters of the silicon solar cells are doped according to the different profiles (erfc, gauss...). These profiles vary according to the depth of the emitter. They are represented by a very high doping level in the front surface $\left(N_{D}=10^{20} \mathrm{~cm}^{-3}\right)$ and a low doping level to the proximity of the junction $\left(N_{D}=10^{16} \mathrm{~cm}^{-3}\right)$ (Fig. $4 \mathrm{~b}$ ). Hence, the mechanism of recombination differs according to the position and the level of doping. On the surface, where the doping level is high, the lifetime is essentially described by the Auger recombination, whereas in the proximity of the junction, where the doping level is moderate, it is described by a mechanism of recombination by trap. Then one adequate modeling of the minority carrier lifetime in the thin emitter must take the two cited mechanisms into account (Fig. 4a). 

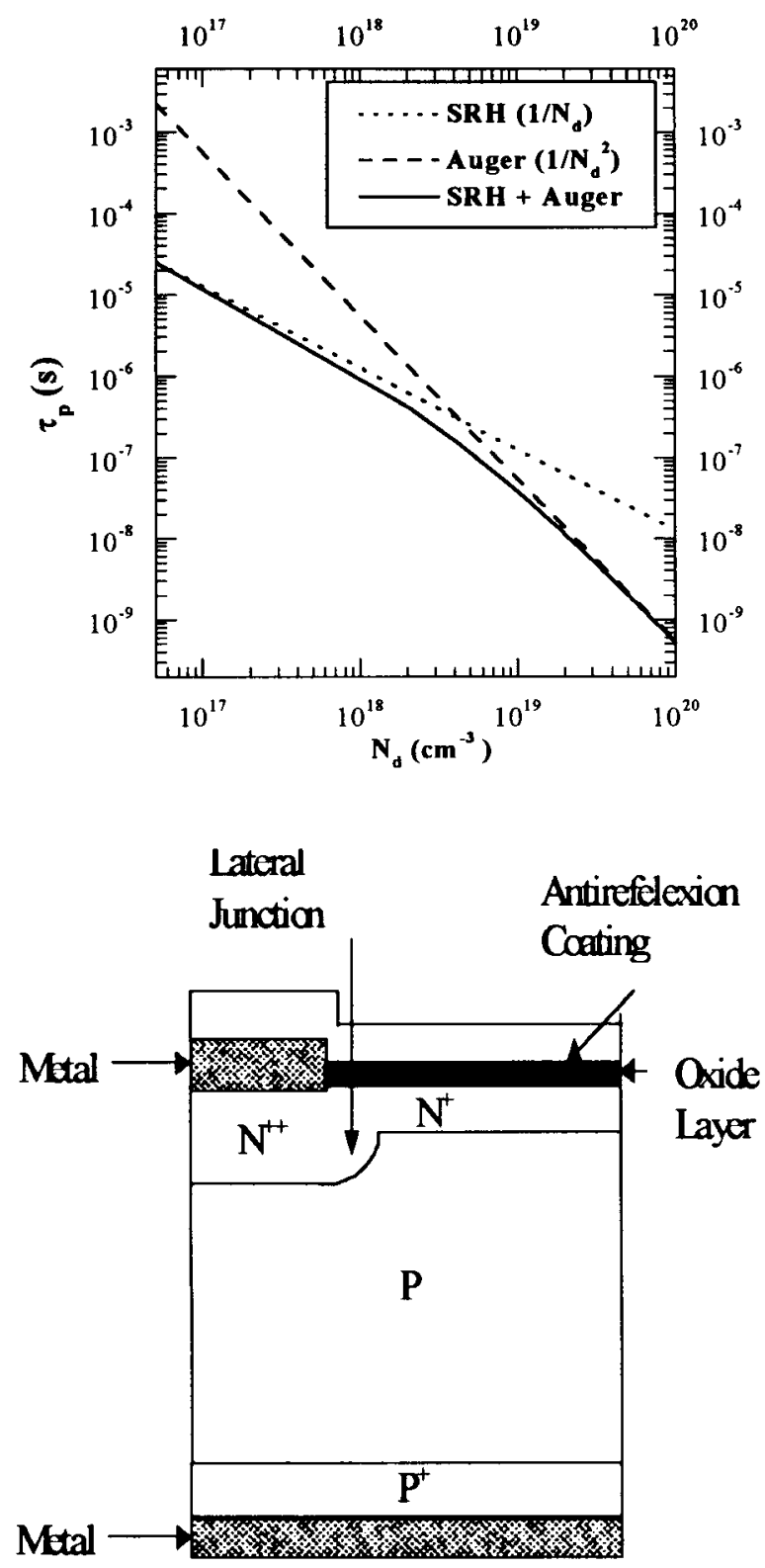

FIGURE 4 a: Representation of the minority carrier lifetime with respect to $N_{D}$ for the two mechanisms of recombination; b: Shames of the $n^{+} p$ considered solar cells.

Del Alamo et al. (1987) have determined the minority carrier lifetime according to the doping level. They proposed a model competitive with all models of the literature. The mathematical expression of this model is composed of two terms. The first depends linearly on the $N_{D}$ doping (recombination by traps) and a second term depends quadratically on the $N_{D}$ (Auger recombination).

$$
\frac{1}{\tau_{p}}=7.8 \times 10^{-13} N_{D}+1.8 \times 10^{-31} N_{D}^{2}\left(\mathrm{~s}^{-1}\right)
$$




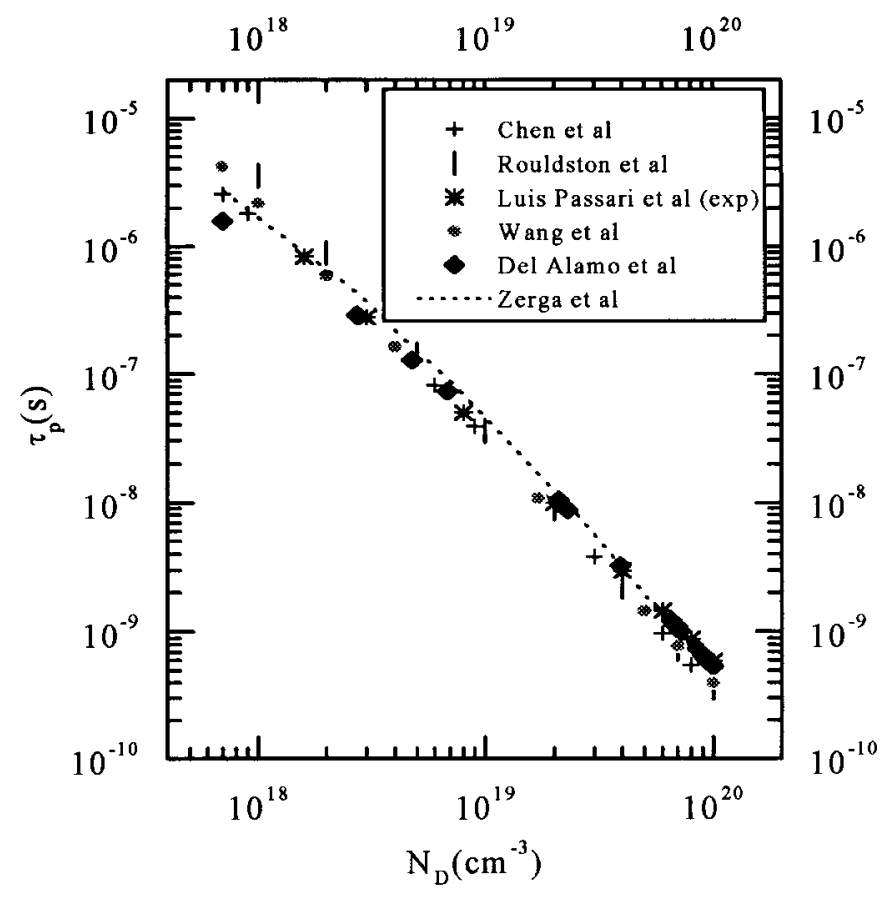

FIGURE 5 Variation of the minority carriers lifetime according to $N_{D}$.

However, we have again developed a numerical program to fit the experimental data of minority carrier lifetime by respect to the doping level by using the model of Green [3]. This model describes the lifetime function by the following expression:

$$
\tau_{p}(\mathrm{~s})=-0.21 \mathrm{~N}_{\mathrm{D}}^{2}+6.46 \mathrm{~N}_{\mathrm{D}}-53.09
$$

Figure 5 represents the variations of the minority carrier (holes) lifetime according to the concentration of donor atoms as well as any different results published in literature.

\section{INFLUENCE OF HEAVY DOPING EFFECTS ON THE OPEN CIRCUIT VOLTAGE AND THE NEW CONCEPT OF THE THIN EMITTER}

Based on the classical theory of Shockley, in the minority carrier transport equation in the heavily doped regions, the effect of heavy doping was neglected. However, to accomplish an adequate simulation of the silicon solar cell with highly doped regions, many authors tried to insert these effects in the general equations of minority carrier transport $[12,15,16]$. They used the effective intrinsic concentration and Auger lifetime. These effects on the performances of the semiconductor devices are considerable. These are due to the distortion of valence and conduction bands. They are also due to the apparition of $\langle\langle$ tails $\rangle\rangle$ band, which affects the recombination, conduction and the drift of the minority carrier in the heavily doped regions. Therefore, the saturation current of the emitter increases and the open circuit voltage decreases. In fact, most attempts to improve the efficiency of the silicon solar cell with a heavily doped emitter were achieved because of the improvement in the open circuit 
voltage. Figure 6 shows the variations of the open circuit voltage according to the thickness $X$ of the emitter.

For values of thickness $X$ below $0.3 \mu \mathrm{m}, V_{\text {oc }}$ depends highly of the surface recombination velocity $S_{f}$ whereas for high values of $X, V_{\text {oc }}$ becomes insensible to the variation of $S_{f}$ and it decreases. It decreases because at high doping level, the thickness of the emitter becomes lower than the minority carrier diffusion length $\left(N_{D}>10^{19} \mathrm{~cm}^{-3}\right)$. Hence, we observe an optimal value of $V_{\mathrm{oc}}$ at the moderate doping level of emitter. This resumes in a very important result where the open circuit voltage of the curve $\mathrm{C}$ is insensitive to the variation of the emitter thickness $X$.

Figure 7 depicts the variations of the open circuit voltage according to the surface recombination velocity.

For values of $S_{f}$ less than $10^{3} \mathrm{~cm} \cdot \mathrm{s}^{-1}$, the open circuit voltage is insensible to the variation of the surface recombination velocity. For greater values of $S_{f}$, the open circuit voltage decreases.

Figure 8 shows the variations of open circuit voltage according to the level of base doping $N_{A}$ for different donor atom concentrations $N_{D}$.

The curves $\mathrm{A}, \mathrm{B}$ and $\mathrm{C}$ show that the high values of $V_{\mathrm{oc}}$ are obtained for doping level varying between $10^{17}-10^{19} \mathrm{~cm}^{-3}$. Beyond these values, the open circuit voltage decreases. This reduction is more important in a thin emitter with a high value of front surface recombination velocity.

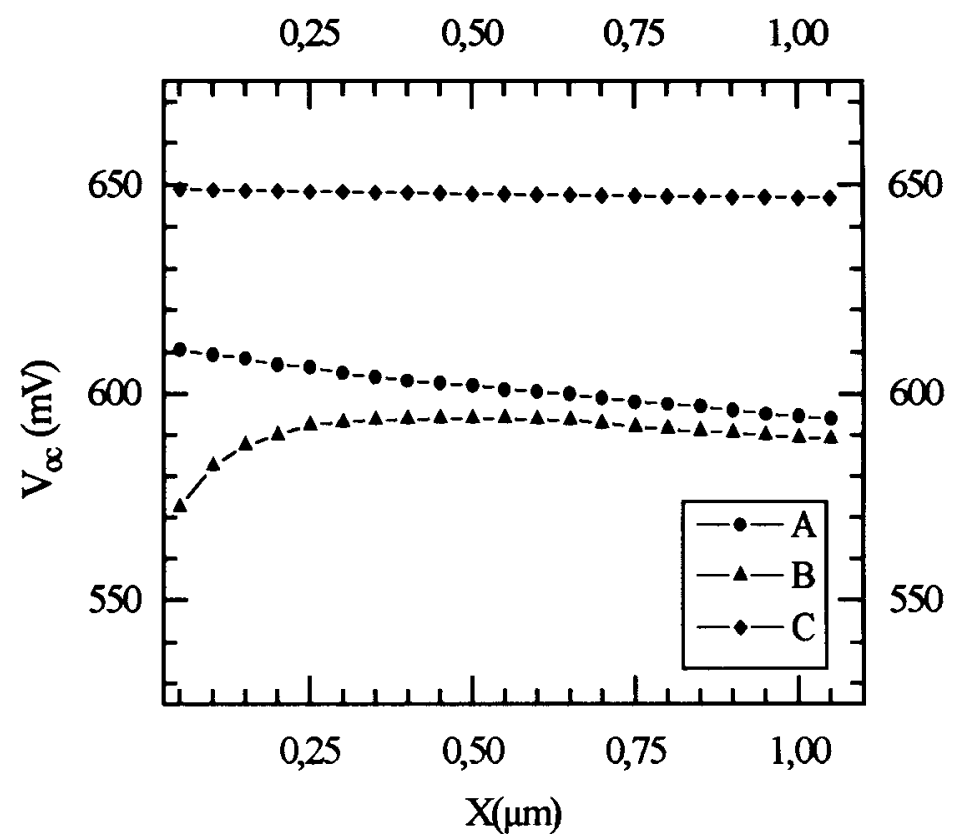

FIGURE 6 Variation of the open circuit voltage $V_{\mathrm{oc}}$ according to the thickness $X$ of the emitter. For curves (A), (B) and (C), we have:

Curve (A): $N_{D}=10^{20} \mathrm{~cm}^{-3}, N_{A}=10^{19} \mathrm{~cm}^{-3}, H=295 \mu \mathrm{m}, S_{b}=10^{5} \mathrm{~cm} \cdot \mathrm{s}^{-1} S_{f}=100 \mathrm{~cm} \cdot \mathrm{s}^{-1}$

Curve (B): $N_{D}=10^{20} \mathrm{~cm}^{-3}, N_{A}=10^{16.5} \mathrm{~cm}^{-3}, H=295 \mu \mathrm{m}, S_{b}=10^{5} \mathrm{~cm} \cdot \mathrm{s}^{-1} S_{f}=10^{6} \mathrm{~cm} \cdot \mathrm{s}^{-1}$

Curve (C): $N_{D}=10^{19} \mathrm{~cm}^{-3}, N_{A}=10^{17.5} \mathrm{~cm}^{-3}, H=295 \mu \mathrm{m}, S_{b}=10^{5} \mathrm{~cm} \cdot \mathrm{s}^{-1} S_{f}=10^{2} \mathrm{~cm} \cdot \mathrm{s}^{-1}$. 


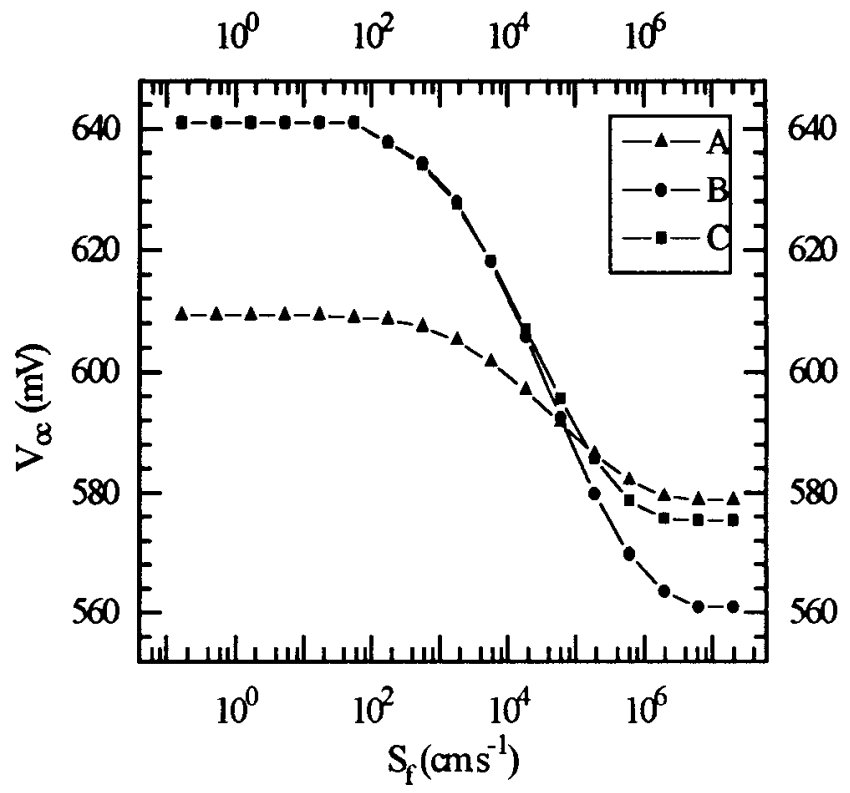

FIGURE 7 Variation of the open circuit voltage $V_{\mathrm{oc}}$ according to the surface recombination velocity $S_{f}\left(S_{b}=\right.$ $\left.10^{15} \mathrm{~cm} \cdot \mathrm{s}^{-1}\right)$. For curves (A), (B) and (C), we have:

Curve (A): $N_{D}=10^{20} \mathrm{~cm}^{-3}, N_{A}=10^{16.5} \mathrm{~cm}^{-3}, X=0.1 \mu \mathrm{m}, H=295 \mu \mathrm{m}$

Curve (B): $N_{D}=10^{19} \mathrm{~cm}^{-3}, N_{A}=10^{17.5} \mathrm{~cm}^{-3}, X=0,1 \mu \mathrm{m}, H=160 \mu \mathrm{m}$

Curve (C): $N_{D}=10^{19} \mathrm{~cm}^{-3}, N_{A}=10^{17.5} \mathrm{~cm}^{-3}, X=0,2 \mu \mathrm{m}, H=160 \mu \mathrm{m}$.

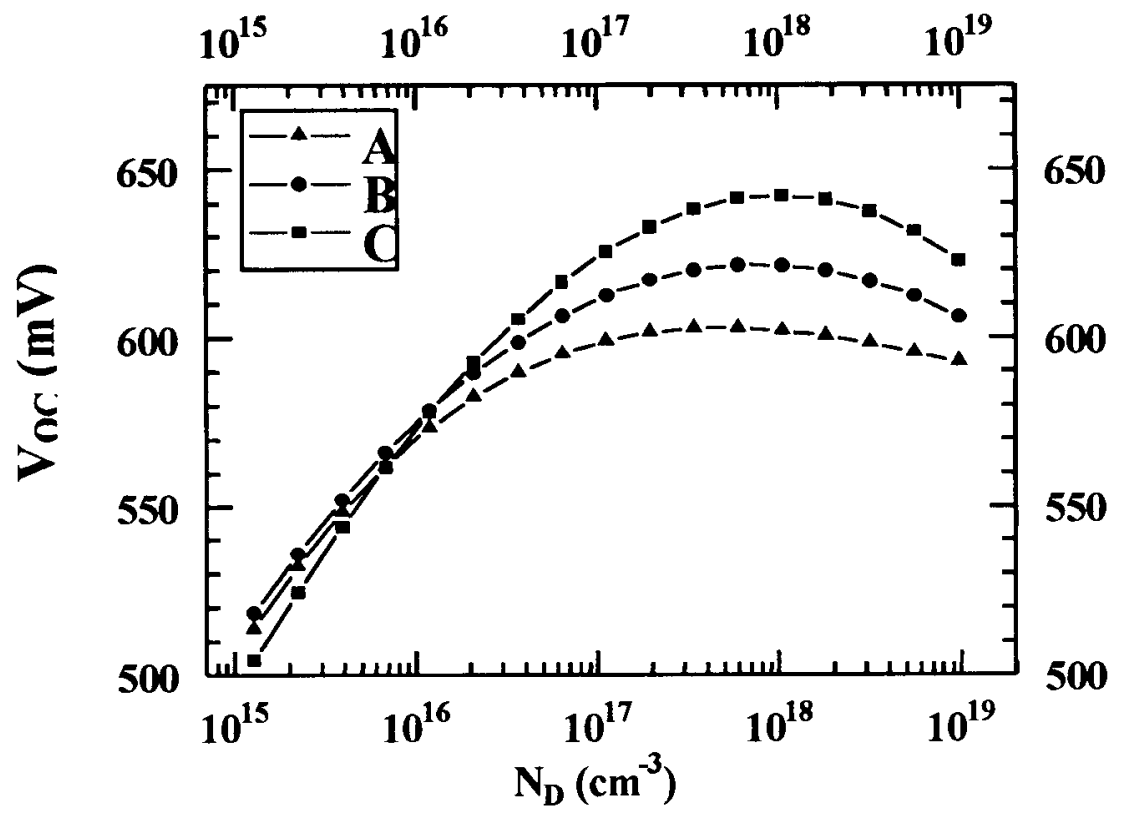

FIGURE 8 Variation of the open circuit voltage $V_{\mathrm{oc}}$ according to the acceptor concentration $N_{A}$. For curves (A), (B) and (C), we have:

Curve (A): $N_{A}=10^{16} \mathrm{~cm}^{-3}, X=0.2 \mu \mathrm{m}, H=295 \mu \mathrm{m}, S_{b}=10^{15} \mathrm{~cm} \cdot \mathrm{s}^{-1}, S_{f}=10^{6} \mathrm{~cm} \cdot \mathrm{s}^{-1}$

Curve (B): $N_{A}=10^{16} \mathrm{~cm}^{-3}, X=0.6 \mu \mathrm{m}, H=295 \mu \mathrm{m}, S_{b}=10^{15} \mathrm{~cm} \cdot \mathrm{s}^{-1}, S_{f}=10^{2} \mathrm{~cm} \cdot \mathrm{s}^{-1}$

Curve (C): $N_{A}=10^{15.5} \mathrm{~cm}^{-3}, X=0.5 \mu \mathrm{m}, H=295 \mu \mathrm{m}, S_{b}=10^{15} \mathrm{~cm} \cdot \mathrm{s}^{-1}, S_{f}=10^{2} \mathrm{~cm} \cdot \mathrm{s}^{-1}$. 


\section{CONCLUSION}

In this study, we have presented a systematic study of the heavy doping effects and their influences on the open circuit voltage of the silicon solar cell with a heavily doped emitter. We have brought some corrections to the coefficients used in the recent models of the band-gap narrowing and the minority carrier lifetime. These corrections have reduced the differences between the theory and experimental data and allow a good simulation of the considered solar cell [17].

The results we obtained show that the open circuit voltage depends on the thickness of the active layer (emitter) and of the doping level $N_{D}$.

A high value of the open circuit voltage can be obtained for a thin and heavily doped emitter, called a transparent emitter or for a thick and moderate doping emitter. In the case of the transparent emitter, a high velocity recombination in front surface can considerably attenuate the open circuit.

\section{References}

[1] Del Alamo, J. and Swanson, R. M. (1987). IEEE Transactions on Electronic Devices, 34, 1580-1589.

[2] Sanjay Rebello, N., Ravipati, C., Zollman, D. A. and Escalada, L. T. (1997). American Journal of Physics, 65, $765-773$.

[3] Green, M. A. (1997). Progress in Photovoltaics: Research and Applications, 5, 261-263.

[4] Bhatnagar, S. and Verma, S. (1997). Indian Journal of Pure \& Applied Physics, 35, 338-343.

[5] Boubekeur, H. (1991). Etude de la région d'Emetteur d'une Cellule Photovoltaïque au Silicium Monocristallin. Thèse de Magister, UDTS Alger.

[6] Slotboom, J. W. and De Graaf, M. C. (1976). Solid State Electronics, 19, 857-862.

[7] Shibib, M. A., Lindholm, F. A. and Therez, F. (1979). IEEE Trans. on Elec. Dev., ED26, 959-965.

[8] Lindholm, F. A., Li, S. S. and Sah, C. T. (1975). 11th IEEE Photovoltaics Specialists Conference, pp. 3-12.

[9] Pan, Y. (1991). Minority carrier transport in heavily doped silicon. PhD, Delft University, Netherlands.

[10] Van Overstraten, R., De Man, M. and Mertens, R. (1973). IEEE Trans. on Elec. Dev., ED-20, 290-298.

[11] Lanyon, H. P. D. (1980). Solar Cells, 3, 289-311.

[12] Del Alamo, J., Swanson, R. M. and Swithurn, S. (1985). Solid-State Electronics, 25, 47-54.

[13] Dziewior, J. and Schmid, W. (1977). Applied Physics Letters, 31, 346-348.

[14] Acedevo, A. (1996). Revista. Mexicana de Fisica., 42, 449-458.

[15] Verhoef, L. A. (1988). Silicon solar cells: modeling, processing and characterization. PhD Dissertation, FOM Institut Voor Atoom - en Molecuul Fysica, Amsterdam.

[16] Pfbeiderer, H. and Bullema, B. (1997). Solar Energy Materials and Solar Cells, 46, 17-27.

[17] Zerga, A. (1998). Optimisation du Rendement des Cellules Solaires à Emetteur Fortement Dopé. Thèse de Magister, Université de Tlemcen. 

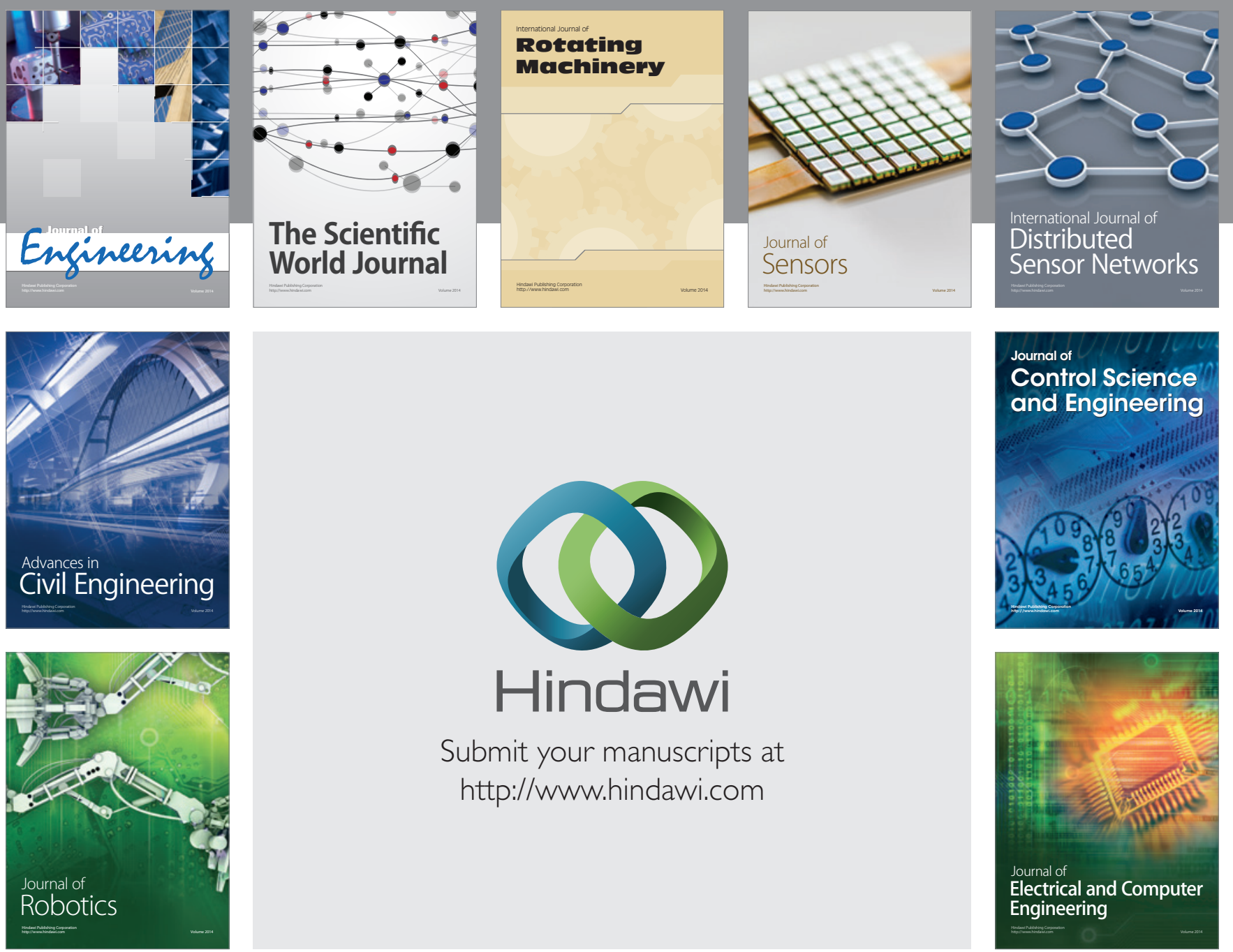

Submit your manuscripts at

http://www.hindawi.com
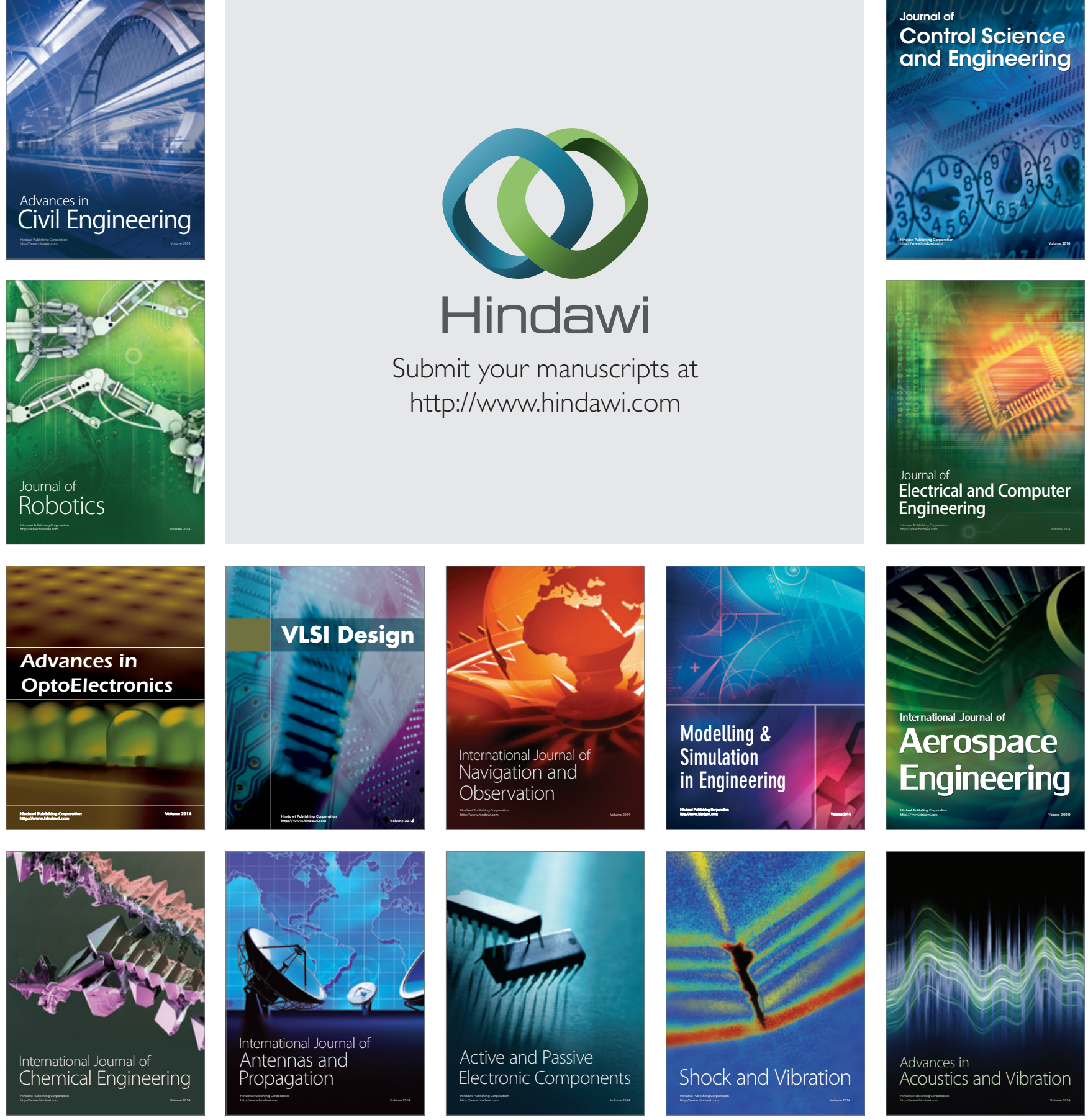